\title{
Medicinal Plants Segmentation using Thresholding and Edge based Techniques
}

\author{
Rajani S, Veena M.N
}

\begin{abstract}
Medicinal value is the integral essence and active ingredients in plants. Knowledge about medicinal plants is more essential to mankind. Manual identification of medicinal plant requires prior information and needs the assistance of botanist because all the plants have similar color, shape and texture characteristics and it is time consuming process. Due to this reason it is very much essential for Pharmacists, Botanist and Ayurveda Practitioners to know how to identify medicinal plants through computer technologies. Image processing technology is used for identification and classification of medicinal plants. The following three general phases of image processing techniques are applied to all types of images namely pre-processing, segmentation and feature extraction. Among all the stages of image processing, segmentation phase partition an image into number of regions and segment the object from background. It improve the representation of an image into more meaningful and easier to analyze the features. The medicinal plants segmentation is carried out in this proposed work. The common and famous segmentation techniques like edge detection and thresholding methods are applied on different shapes of flowers, leaves and fruits/seeds images. This paper explores the comparisons of above two methods and effects of filtering and enhancement techniques.
\end{abstract}

Keywords - Edge, Images, Segmentation, Threshold.

\section{INTRODUCTION}

Plants species are becoming extinct because of global warming, increase in population growth and lack of awareness of medicinal plants. Identification of herbal and nontoxic plants is considered as important activity for Pharmacy Graduates, Botanist, Research Students, Ayurveda Practitioners, Pharmaceuticals companies and Herbal Researchers. This process consume more time and one should have prior knowledge about medicinal plant identification. With the help of information technology and lot of innovation in the computer field, image processing is used in identification and classification of medicinal plants species. Digital image processing converts an image into digital form and perform operation such as create, process and improves the picture quality. The basic steps in digital image processing is Image Acquisition, Enhancement and Restoration, Morphological Processing, Segmentation, Representation and Description and Object Recognition. Image segmentation is the important part of image processing which segments or partitioning an image into meaningful areas according to some characteristics such as gray level, spectrum, texture, and color. These features are very much needed for identifying medicinal plants. Some of the methodologies uses both input and output as images. In segmentation methods inputs are images and outputs are attributes extracted from those images.

\section{SEGMENTATION}

Images are divided into different non overlapping regions or parts and this separate the objects from background region. The main stages of segmentation is removal of unnecessary information, identification of object of interest [1] and making easier to identify features. The characteristics of image segmentations are regions, edges, area, perimeter, statistics and entropy [2].

$\mathrm{R}$ represents the spatial region of the image. Partition $\mathrm{R}$ into $\mathrm{n}$ sub regions, R1, R2...Rn. The similar region pixels are partitioned with respect to either color, intensity, or texture. Segmentation techniques are classified into two main categories, layer-based segmentation methods and block-based segmentation methods [6]. In this study only block based segmentation type is considered. This segmentation method uses the following two properties, Similarity and Discontinuity. The similarity approach partitions an image into regions where as discontinuity property partitions an image into edges based on abrupt changes in intensity. Following predefined conditions are needed to partition an image, (a) all the pixel points must 4 or 8 connected and should be in regions, (b) the adjacent regions must be disjoint. Segmentation algorithms are as follows Thresholding (similarity), Edge based segmentation (Discontinuity), Hough transform (Discontinuity), Region based segmentation (similarity), Watershed segmentation (Discontinuity), Match Based Segmentation (Similarity), Artificial Neural Networks and clustering (Similarity). These methods are aimed at specific problems but not suitable for all images [3].

This paper demonstrate the segmentation of medicinal plants using Global and Local Thresholding methods and Edge based first order derivatives methods. The following section III and IV will give the overview of the above mentioned segmentation techniques and followed sections will give the experimental results and pros and cons of these techniques.

\section{THRESHOLDING SEGMENTATION}

Thresholding technique converts a gray sale image into a binary image with the help of histogram. Histogram is the graphical representation of a gray scale distribution in the digital image and shows the number of times each gray level occurs [4]. Background and foreground of the intensity of the image is spread over the histogram. The intensity levels of the foreground object is different from the background. 
Based on the threshold value, histogram techniques separates the twoidentical regions [5]. Determine a threshold value $\mathrm{T}$ for each image pixel and it is computed with the aid of the image histograms peaks. Threshold value $T$ is obtained based on the gray value of different objects [6]. If object of the image pixel is greater than threshold value $\mathrm{T}$ is called as foreground object otherwise background object. The original image $f(x, y)$ and segmented image $g(x, y)$ is given in the following equation (1),

$$
g(x, y)=\left\{\begin{array}{l}
1, \text { if } f(x, y)>T \\
0, \text { if } f(x, y) \leq T
\end{array}\right.
$$

When $\mathrm{T}$ is constant over an image, the process is referred as global threshold. If $\mathrm{T}$ changes on local characteristic of the image, the process is referred as variable threshold. Following are the few of the variable and global threshold methods are considered in the proposed experiment. Otsu's global threshold method find the optimal value at binarization level where as iterative algorithm perform well in global thresholding if the clear valley is present in the two modes of the histogram. Further variable threshold is divided into two types, if the threshold value is associated with the gray level of each pixel (x,y), it is called local threshold. If the selected threshold value is not associated with each pixels but related to the location of the pixel, then it is called dynamic threshold or adaptive threshold. In multiple thresholding two threshold values $\mathrm{T} 1$ and $\mathrm{T} 2$ are considered. The segmented image $\mathrm{g}(\mathrm{x}, \mathrm{y})$ in equation (2) is given by with respect to multiple thresholding,

$$
g(x, y)=\left\{\begin{array}{c}
1, \text { if } f(x, y)>T 2 \\
1, \text { if } T 1<f(x, y) \leq T 2 \\
1, \text { if } f(x, y) \leq T 1
\end{array}\right.
$$

Threshold segmentation process having different threshold values. If the threshold value is large, it will segment the background pixels into many targets objects. If it is small, it will segment target pixels into backgrounds. If there is less noise in image, threshold segmentation can be determined easily. Image thresholding is most effective for high contrast images. Table 2 illustrate the thresholding techniques are applied on medicinal plant images which are corrupted by Gaussian noise as well as Salt and Pepper noise.

\section{EDGE DETECTION METHOD}

Image object are segmented from its background based on variations in intensity level [8]. The variations in the black and white intensities forms the edges in the object. First identify the closed boundaries of different regions then segment the pixel that have closed connect boundaries. Edge detection method converts image into gray, then before detecting any edges it removes the noise in the image, this process is called filtering or smoothing. After filtering and enhancement of the image, Edge Detection operator is applied which is illustrated in Fig 25. The categories of edge based segmentations are listed below. Discontinuity property is detected by these categories. The proposed work considered the First order derivatives operators which is illustrated in table 3 . The prime choice of first order derivative is image gradient. The gradient vector of the image is depends on the direction in which intensity changes at the pixel points.

\section{First order derivatives operator: \\ 1. Prewitt operator \\ 2. Canny operator \\ 3. Sobel operator \\ 4. Roberts operator \\ 2. Second order derivatives operator:
1. Laplacian of Gaussian (LoG)
2. Gaussian Edge detector
3. Colored edge detector

\section{IMPLEMENTATION\& RESULTS}

Different shapes and texture of medicinal plants images are chosen for Experiment. Following tables illustrate the applications of different segmentation methods. To improve the visualization of the blurred images and noised images, different enhancement techniques and filtering techniques are used in table 2 and table 4. Many Filtering and Enhancement techniques are available to make haze free images. In this paper few of the techniques are considered for removing blur and noise in the images.

Table 1 demonstrate the comparative analysis of both Otsu Thresholding method and Adaptive Thresholding methods applied on similar kind of background of Rose images which are vary in color and contrast.

In table 2, Salt and Pepper noise and Gaussian noise is applied on original images of Lotus flower and Lime fruit images respectively. Otsu Global Thresholding method is applied on both noised images and filtered images. The comparative analysis of before and after filtering images of thresholding are shown from Fig. 5 to Fig. 14. Following Filtering techniques are applied on noised images such as Average filter, Median filter, Arithmetic Mean filter and Wiener filter.

Table 3 illustrate the Edge Detection operators such as Canny, Sobel, Prewitt and Roberts operators. These operators are applied on different textured images namely, Calotropis flower, Betel leaf, Hibiscus flower and Amla fruit images. The comparative study shown from Fig. 17 to Fig. 22.

Blurred Tulasi leaf image is considered in Fig 23. To enrich image visualization, following Enhancement methods like Contrast Adjustment techniques, Histogram Equalization and Adaptive Thresholding Equalization techniques are applied on blurred image which is shown in Fig. 24. After Enhancement, Canny Edge operator is applied on the Enhanced image which is illustrated in Fig 25.

\section{ADVANTAGES AND DISADVANTAGES}

Threshold methods and Edge detection methods yield best result with grayscale images having fine features of flowers and leaves. Gray images are combination of black and white color. Black color have low intensity where as white color have high intensity information. 
The proposed work illustrate the Threshold methods yield better result on different background of Rose images and Lotus images but fail to yield results on Lime fruit image because of lack of texture features. Edge Detection methods perform well on fine features of Hibiscus flower and Betel leaf images but fail to yield results on Calotropis flower and Amla fruit images because of lack of texture features.

Threshold methods are not suitable for flat portion and images with too many edges. In Edge Detection methods, if edges are clearly defined, Canny Edge Detection method work well on textured flower and leaf images but if edges arenot defined properly and images are edgeless and texture less Edge Detection algorithm won't work well.

Experiment demonstrated, the performance of both Adaptive Thresholding and Otsu Global thresholding segmentation methods not yield best results on images with high contrast from background. Median filter and Wiener filter yield better result on Salt and Pepper noise and Gaussian noise images respectively. Adaptive Histogram Equalization method yield better result on blurred image compare two other three Enhancement techniques.

Table 1.Thresholding Methods

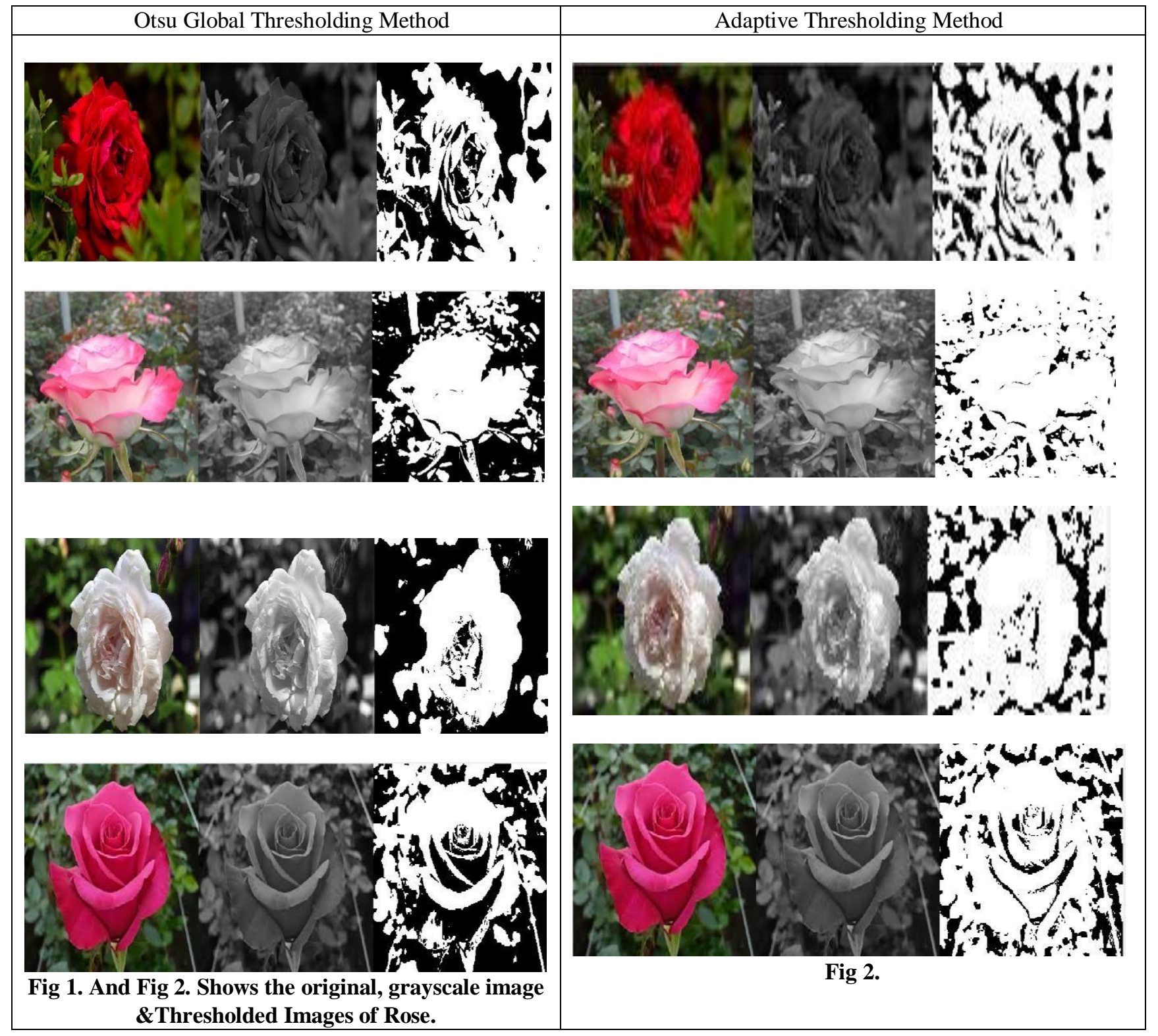

Table 2. Filtering and Otsu Thresholding Methods on noised Images.

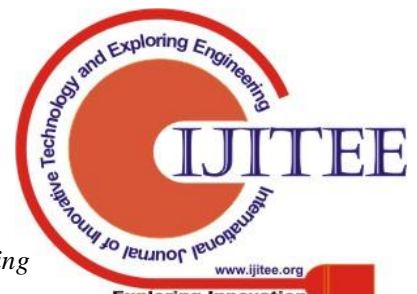




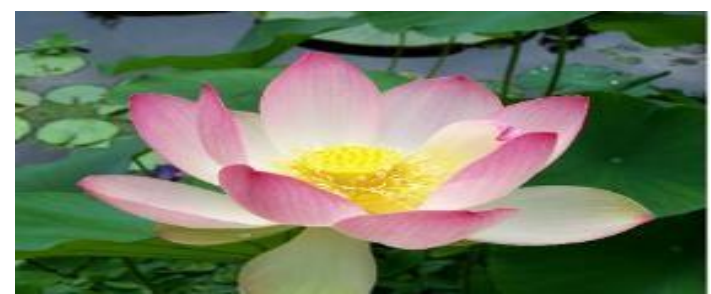

Fig 3. Lotus flower.

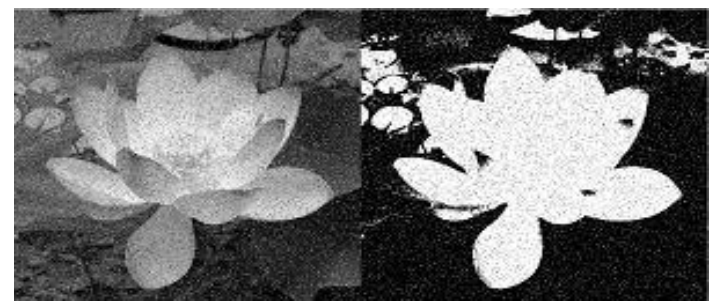

Fig 5. Salt \& Pepper noise image and Thresholded image with Noise.

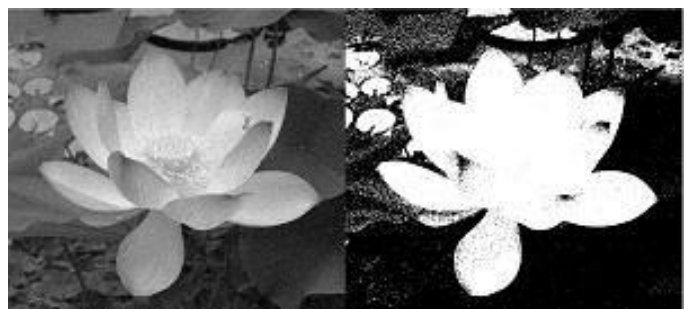

Fig 7. And Fig 8. Average Filter image And Thresholded images.

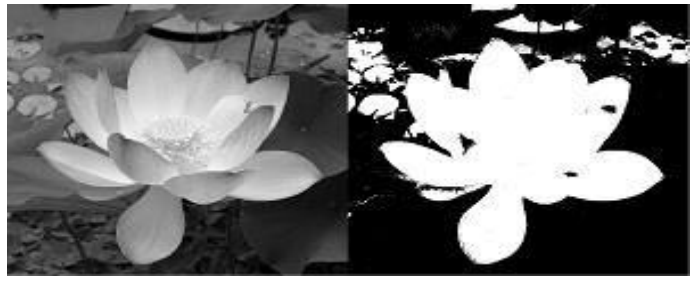

Fig 9. And Fig 10. Median Filter image And Thresholded images.

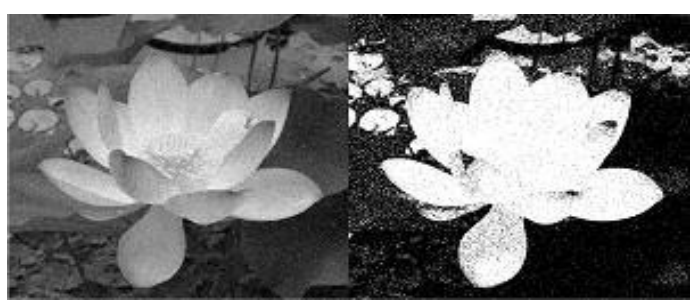

Fig 11. And Fig 12. Arithmetic Mean Filter images and Thresholded images.

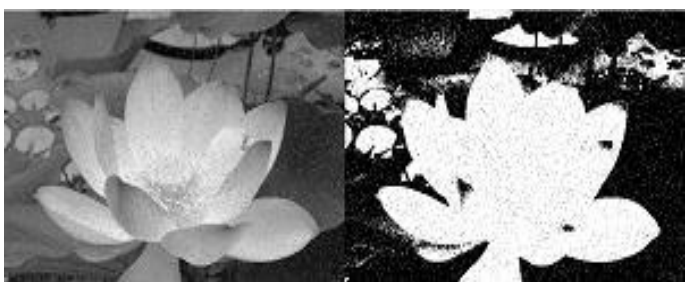

Fig 13. And Fig 14. Shows Weiner Filter images and Thresholded images.

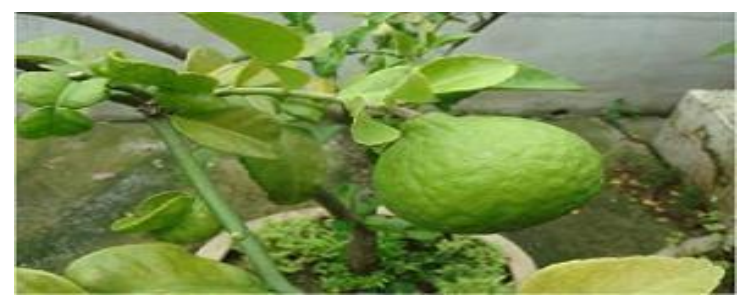

Fig 4. Lime fruit image.

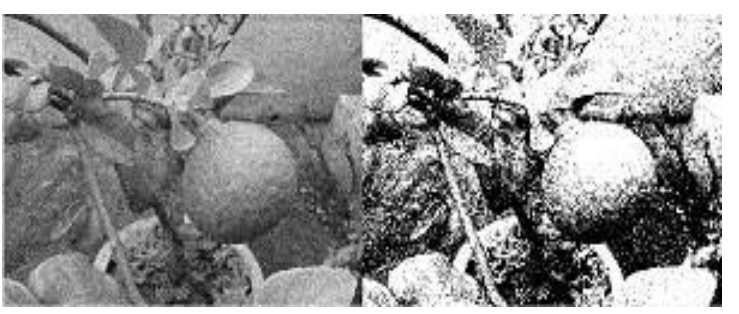

Fig 6. Gaussian noise and Thresholded image with Noise.

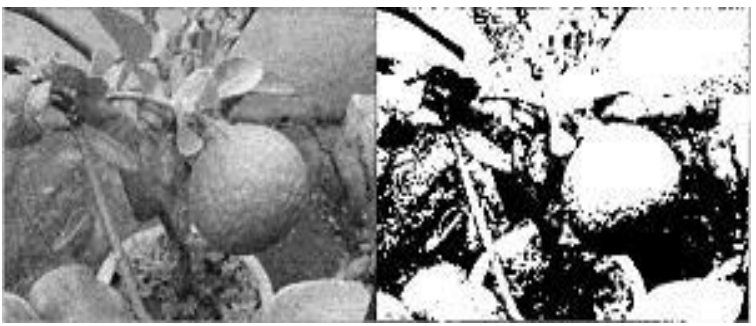

Fig 8.

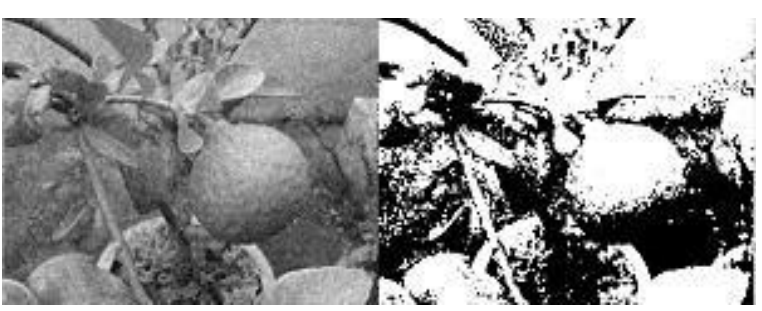

Fig 10.

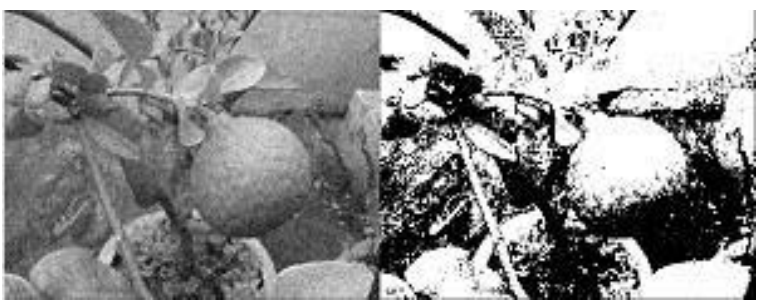

Fig 12.

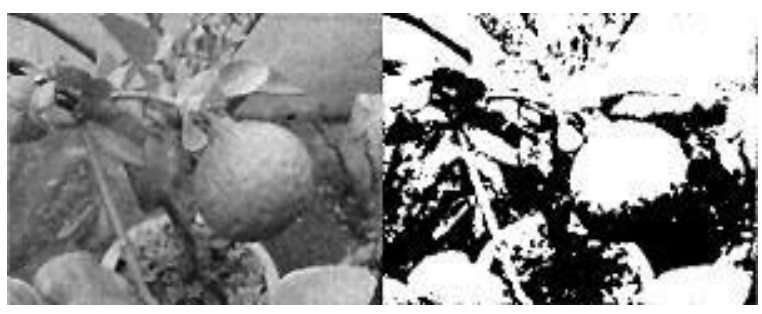

Fig 14.

Table 3. Edge Detection Methods: First order derivatives operators.

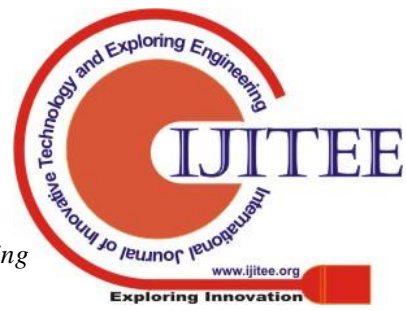




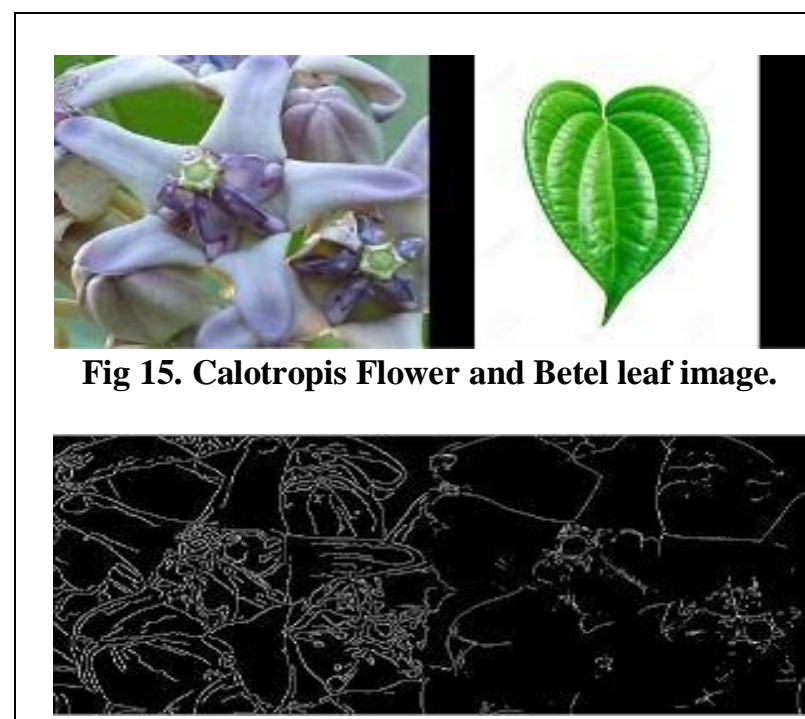

Fig 17. And Fig 18. Canny and Sobel edge operators images.

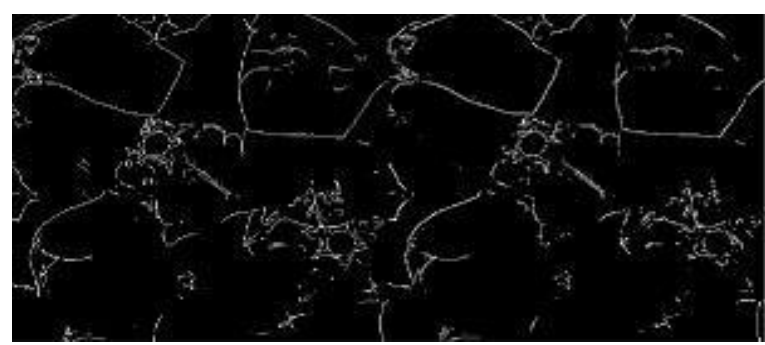

Fig 19. And Fig 20. Prewitt and Roberts Operators images.

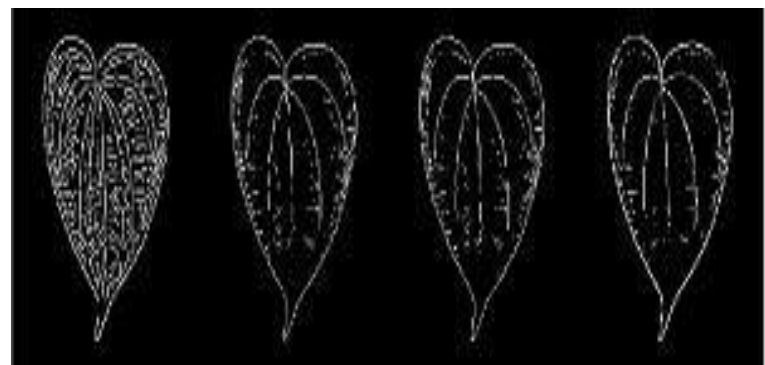

Fig 21. And Fig 22. Canny, Sobel, Prewitt \& Roberts operators images.

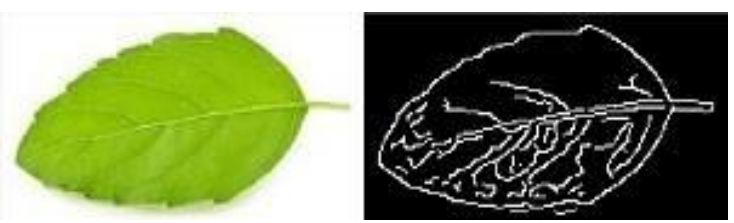

Fig 23. Blurred Tulasi leaf image with Canny edge detection (Before Enhancement technique).
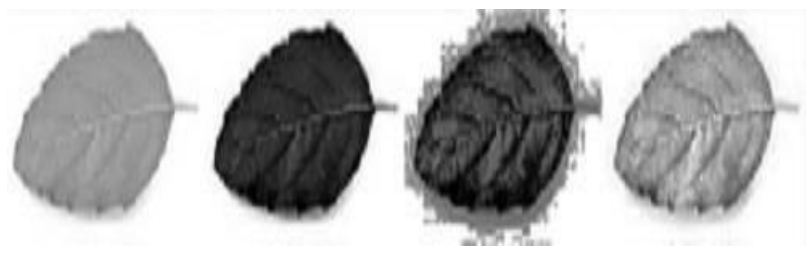

Fig 24. Grayscale Image, Contrast Adjustment

method, Histogram Equalization method and Adaptive Histogram Equalization method.

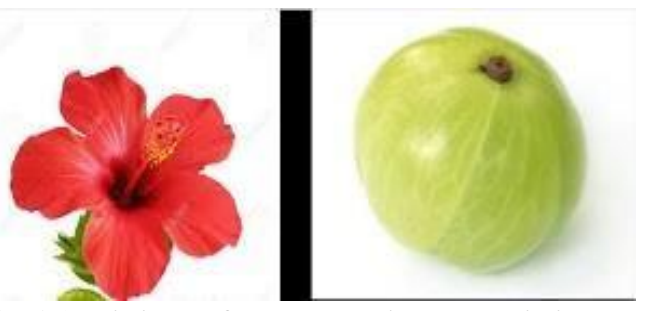

Fig 16. Hibiscus flowerand Amla Fruit image.

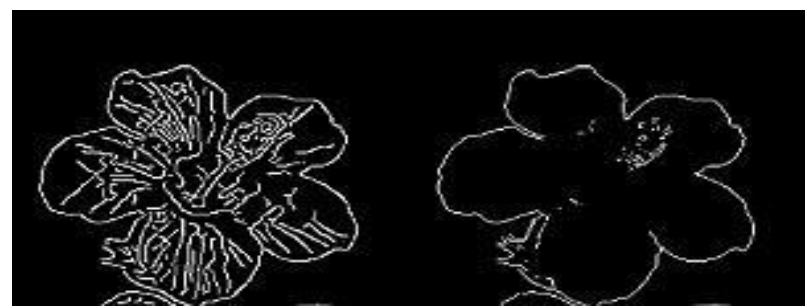

Fig 18.

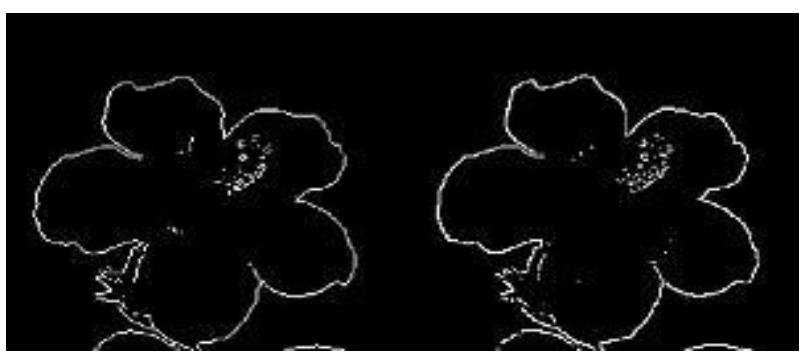

Fig 20.

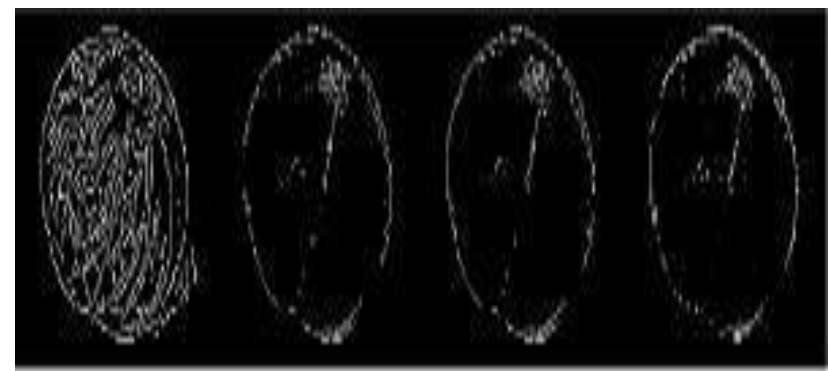

Fig 22.

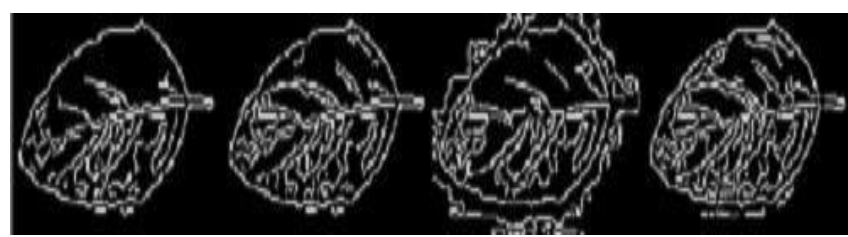

Fig 25 Canny edge detection methods after Enhancement Technique.

\section{CONCLUSION}

The proposed work explains the segmentation of different background of medicinal plants images using segmentation methods. Since there is no general solution to the medicinalplants image segmentation problem because images are in different shapes and textures and also the impact

of

noise

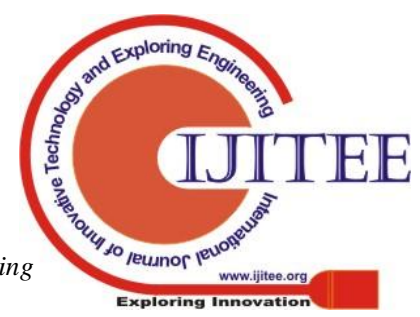


and brightness of the images. From proposed experiment, the efficiency of Edge Detection methods and Thresholding methods is not up to the mark for different shapes and texture of flower, leaves and fruits images of medicinal plants. The proposed work suggest the hybrid techniques will effectively solve image segmentation problem than applying individual methods. The challenging task is to identify which method is the most suitable for a particular shape of the images. Further research work will improve the result by using hybrid segmentation techniques on particular data set.

\section{REFERENCES}

1. Nida M, ZaitounaMusbah J, Aqelb, "Survey on Image Segmentation Techniques," International Conference on Communication, Management and Information Technology (ICCMIT 2015).1877-0509@ 2015 Published by Elsevier.

2. KinjalMunot, Nishi Mehta, Sakshi Mishra, Ratnesh N, Chaturvedi, "A Review on Image Segmentation Techniques with an Application Perspective," Volume 8, No. 9, November-December 2017. IJARCS.

3. Rafael, C., Gonzalez, Richard, E., Woods. : Digital Image Processing. University of Tennessee, MedData Interactive. (C) 2008 by Pearson Education, Inc.

4. Naveen Tokas, ShrutiKarkra, Manoj Kumar Pandey, "Comparison of Digital Image Segmentation Techniques- A Research Review," International Journal of Computer Science and Mobile Computing, Vol.5 Issue.5, May- 2016, pg. 215220.

5. Rahul Basak, Surya Chakraborty, Aditya Kumar Mondal, SatarupaBagchiBiswas, "Image Segmentation Techniques: A Survey," Volume 05 Issue. 04 |Apr-2018, International Research Journal of Engineering and Technology (IRJET).

6. Song Yuheng, Yan Hao, "Image Segmentation Algorithms Overview".

7. C Mariyammal, S Sasireka, "Survey on Image Segmentation Methods," International Advanced Research Journal in Science, Engineering and Technology. Vol. 5, Issue 11, November 2018.

8. Dharmendra Kumar, Kanak Kumar, "Review on Different Techniques of Image Segmentation Using MATLAB," International Journal of Science, Engineering and Technology. 2017, Volume 5 Issue 2.

9. C Ananthi, Azha, Periasamy, S Muruganand, "Pattern Recognition Of Medicinal Leaves Using Image Processing Techniques," Journal of NanoScience and NanoTechnology, Volume 2, Issue 2, Spring Edition, ISSN 2279 - 0381.

10. I KirubaRaji, K KThyagharajan, "An Analysis of Segmentation Techniques to Identify Herbal Leaves from Complex Background," ICCC 2014, 1877-0509 @ 2015, Published by Elsevier B.V.

11. PatilPriyanka Vijay, Prof N. C. Patil, "Gray Scale Image Segmentation using OTSU Thresholding Optimal Approach," Journal for Research, Volume 02, Issue 05, July 2016.

12. PinakiPratimAcharjya, Ritaban Das, DibyenduGhoshal, "A Study on Image Edge Detection Using the Gradients," International Journal of Scientific and Research Publications. Volume 2, Issue 12, December 2012.

13. Mohd. Aquib Ansari, DikshaKurchaniya, Manish Dixit, "A Comprehensive Analysis of Image Edge Detection Techniques," International Journal of Multimedia and Ubiquitous Engineering, Vol.12, No.11 (2017), pp.1-12.

14. Fari Muhammad Abubakar, "Study of Image Segmentation using Thresholding Technique on a Noisy Image," International Journal of Science and Research (IJSR), India Volume 2 Issue 1, January 2013.
15. EsmaelHamuda, Martin Glavin, Edward jones, "A survey of image processing techniques for plant extraction and segmentation in the field," Computer and Electronics in Agriculture 125(2016)184-199.

16. S.S. Lomte1, A.P. Janwale,'Plant Leaves Image Segmentation Techniques: A Review", Volume-5, Issue-5, E-ISSN: $2347-$ 2693. 